\title{
Implementation of Absolute Priority in a Predictive Traffic Actuation Schemes
}

\author{
Souhir Boudhrioua ${ }^{1 *}$, Mohamad Shatanawi ${ }^{1}$ \\ 1 Department of Transport Technology and Economics, Faculty of Transportation Engineering and Vehicle Engineering, \\ Budapest University of Technology and Economics, H-1111 Budapest, Múegyetem rkp. 3., Hungary \\ * Corresponding author, e-mail: souhir.boudhrioua@gmail.com
}

Received: 10 April 2019, Accepted: 08 July 2019, Published online: 14 November 2019

\begin{abstract}
Nowadays, the improvement of the emergency vehicles' priority is required to improve their response time. Presently, the emergency vehicles alert the road users using the sirens and lights, which might cause disturbances in the traffic or lead to accidents due to the unpredictability of the road users' behaviors. This paper introduces the priority indicator, which calculates the priority of several emergency vehicles as they approach an intersection and sort them in a way that allows granting priority to each one without causing delays neither for the emergency nor for the normal vehicles. The priority indicator is then introduced in a predictive traffic actuation program (DIRECTOR) that adjusts the signal timing in a dynamic way. Thus, this research aims to create a generalized priority form that simplifies granting the priority for emergency vehicles in a harmonized way in all over the Netherlands, making sure that it takes into consideration the several conditions of the intersection.
\end{abstract}

\section{Keywords}

emergency vehicle, priority, traffic congestion

\section{Introduction}

Worldwide, traffic congestion comes with significant external costs due to additional travel time, wasted fuel and increase in traffic accidents (Bigazzi and Figliozzi, 2013). Numerous measures can be taken to address problems due to traffic congestion. Therefore, operators and planners seek to create and implement the ability to estimate and forecast traffic conditions with appropriate accuracy and reliability (Vlahogianni et al., 2014).

In previous works with Siemens Mobility Zoetermeer, NL, a traffic controller having the ability to predict the coming traffic and generate a fixed ahead schedule for the coming 10s vehicles has been made, giving the possibility to deal with the congestion problems in a variable adaptive way based on the intersection's conditions. The model is dealing with complex, conflicting and dynamic data, but even though it is of a great importance, it needs to be more adaptable in a way that it takes into consideration the different types, situations and target response-time of the coming vehicles. Thus, the aim is to implement the priority factor for emergency vehicles, and expectantly in coming researches, for the various vehicle's classes; trucks, buses, trams, bicycles, etc., in order to make sure that all traffic is handled in the best way (van Senden, 2018).

Different research projects proposed multiple approaches to give priority for emergency vehicles (EVs) such as the use of green wave (Viriyasitavat and Tonguz, 2012) or priority based only on the distance of the EV from the intersection (Nellore and Hancke, 2016) or on the assumption that the OD (Origin_Destination) is known from the start of the vehicle (Kapileswar et al., 2017). Despite this, most of the researches do not deal with the situation in a dynamic way or consider that only one emergency vehicle is approaching from one direction at a time. Moreover, most research projects find it hard to classify the EVs (ambulance, fire truck and police) because it involves an ethical perspective, so usually the priority is provided to an ambulance first, however, real life scenarios are much more complicated. In case of a fatal accident, multiple EVs face crucial response timing and generally need to attend the scene through different paths from different locations.

Often EVs are lawfully entitled to break conventional traffic rules such as crossing an intersection in the red 
signal light or exceeding the speed limit to reach their destinations in the shortest time possible. Yet, this might be extremely dangerous for the EVs themselves, as they can get involved in accidents as well.

In this paper, motivated by the previously mentioned dilemmas, a new approach to grant priority for emergency vehicles is introduced. Using a tool that can predict a certain number of EVs driving towards an intersection, this work seeks to give EVs priority based on the Estimated Time of Arrival (ETA), the Time to Dissipate the queue $\left(t_{d}\right)$ and the priority class. These factors are used to measure the Priority Indicator (PI) that shall be implemented in the traffic actuation program (Data-driven Intersection and Road Environment Controller for Traffic Optimization in RealTime- Director). This target, on one hand; to make it possible for different EVs to reach the accidents' destinations in the safest way without exceeding their response target time. On the other hand, to keep the traffic moving as smooth and safe as possible without giving long unneeded red time.

\section{Methodology}

The dynamic traffic signal based on priority allocation is the future of the Traffic Light Controllers (TLC), which would allow the granting of priority for emergency vehicles when needed without causing traffic congestion and assuring a safer circulation. First of all, the different parts of the environment where the PI is going to be implemented and their relationships is realized. Second part is dedicated for the literature review and the lessons learned which would help understand the background of the inspired Priority Indicator. The last part is concentrated on the procedure of how the priority can be allocated through the PI and its variation in different scenarios.

\section{Background}

The priority indicator is meant to be integrated in the system DIRECTOR and taking into consideration the road systems in the Netherlands. Thus, the environment in which the priority indicator is going to be integrated is described in the following parts.

\subsection{Introduction of DIRECTOR}

DIRECTOR is a patent pending distributed predictive TLC, developed by Noorhan Helmy and adjusted by Jan Cees van Senden within Siemens Mobility SNLM. It uses the data produced by the stopline detectors from the upstream intersections to predict the coming flow to the downstream one. The flow is adjusted with looking into the data from the arrival, queue and stopline detectors of the intersection itself (Helmy, 2017).

DIRECTOR is the acronym for the Data-driven Intersection and Road Environment Controller for Traffic Optimization in Real-Time. It helps handling the traffic lights in a predictive and real-time optimized way (Helmy, 2017).

Yet, it does not distinguish between the traffic mix, thus all vehicles (EVs, trams, motorcycles, buses, etc.) have the same weight $(=1)$. While this is a primordial tool for an optimized traffic flow and especially when an emergency vehicle is present within the flow, but it is seen as a simple vehicle unit, which can generate a frustration among the road users and a chaos in the traffic if not taken into consideration in the schedule implementation. This created the motive to study how a weighting can be implemented in DIRECTOR (van Senden, 2018).

\subsection{Priority classes}

In the Netherlands, the priority policies are different from one municipality to another and they are based on several criteria such as the vehicle type, the threshold values, besides priority granting is different per road owner and even per intersection which makes it unclear and nontransparent. As a consequence, a uniform universal language for control program has been created in the Dutch Profile to solve the dilemma and it enables vehicles to get priority national wide in the same way. Besides, the priority classes have been introduced and they are referred to as "Prio klass $k$ " such as $k \in[1,14]$, where 14 is the highest priority and 1 is the lowest one.

The priority classes are a part of granting the priority that is realized by the road owners, but the priority classes alone do not allow giving a uniform application of priority in the whole country, therefore, other parts and functions ought to be included in order to handle the priority grating (Logghe et al., 2017).

\subsection{Parts involved}

Granting the priority is a process involving several parts, which are summarized in the Fig. 1.

- Priority Broker Configuration (PBC): it helps the road owner build the priority rules based on a set of policies.

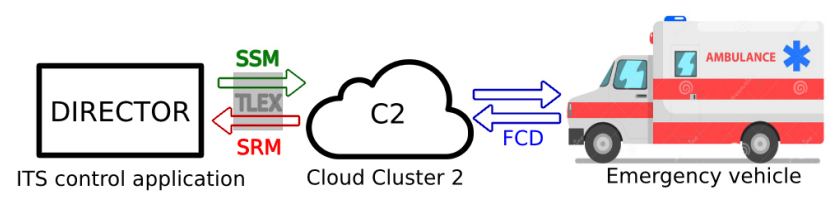

Fig. 1 Relationship between the responsible parts for priority granting 
- Cloud service (C2): Siemens Mobility NL developed the Cluster 2 (C2) and it is used as a position-based service, it receives information of the moving vehicle on the grid. Thus, it has the ability to determine the distance of the vehicle to an intersection, its speed, its type so it can determine the ETA (Estimated Time of Arrival) of the emergency vehicle. Besides, the $\mathrm{C} 2$ knows at any iVRI (iVRI viewer) what is the priority class accorded to each vehicle type and it is able to know if a vehicle moving on the grid is an EV or not and if it has a flashing blue light and siren or not. As a result, the $\mathrm{C} 2$ helps to even give priority to an emergency vehicle that is moving towards an accident location without/before the EV requests a priority.

- The ITS application DIRECTOR previously introduced in Subsection 3.1 (Logghe et al., 2017).

Normally, the C2 sends CAM messages (Cooperative Awareness Message) to DIRECTOR that include information about the vehicles (vehicle ID, speed, position, as the Intersection ID, type of vehicle, ETA) in an intersection or going to it. When a vehicle requests priority or the $\mathrm{C} 2$ intend to give priority to a vehicle based on its condition, an SRM message is sent from the C2. The SRM is like the CAM message but with a request for service (priority) and when sent from the $\mathrm{C} 2$ to DIRECTOR it contains information about the requested service and the vehicle: the type of vehicle asking for the priority as well as the level of importance of the request.

In an SRM the position of the vehicle is not always delivered because sometimes it is confidential like the example of the police. However, as the vehicle ID should be the same in both the SRM and CAM messages then, it can be deduced as the $\mathrm{C} 2$ received previously the FCD (Floating Car Data) from the vehicle including its position.

The SSM (Service State Message) is the response for the SRM (Service Request Message) and it can be an approval or a message whether providing a priority is possible or not or if the application is still processing.

The TLEX is just a tool that helps to transfer the SRMs and SSMs to the right receiver

\section{Related works}

4.1 Priority and secured system for EVs by the use of IoT Kapileswar et al. (2017) introduced an ITS for emergency vehicle linked to infrastructure (EV2I) considering the priority of an emergency vehicle depending on the type of an incident. The paper proposes an ITS solution of granting priority to EVs and compare it with the base and the hacked situation - the authors take into consideration that the system can be hacked which causes congestion and delays that need to be prevented- in the current operating system, green wave system and emergency priority code system. The implemented classification of the priority of the three EVs (Fire truck, Ambulance, Police) involved in an accident based on three scenarios of accidents.

A dynamic traffic control process is used where the central traffic collects data about the condition of the intersection and calculates the distance $d$ of the flow based on the number of cars $n$, the length of cars $k$ and the distance between two consecutive cars $m$, this is summarized in Eq. (1):

$d=n k+m(n-1)$.

In this work, it is assumed that the OD (OriginDestination) of the EV is known, so the first signals will start operating from the moment the EV leaves its origin. Then, the green wave concept is used to turn green the traffic lights of the downstream intersection, as soon as it leaves the upstream intersection, in order to release all the vehicles in front of the emergency vehicle.

Even though it is not always advisable to use the green wave concept, but in this paper, it is justified as it helps clear the way in front of the EV.

\subsection{Frequency and Distance based Priority}

Chowdhury (2016) proposed an information dissemination system based on a new MAC protocol named FDPMAC (Frequency and Distance based Priority) in order to send emergency messages to the center of traffic management and to other vehicles with less delays. Thus, a Radio Frequency Sensor Units (RFSUs) is utilized as well as Signal Aggregators (SAs), Signal Stations (SSs). The developed traffic systems include light emitters, Infrared (IR), Global Positioning System (GPS) and RFID tags for EV presence detection.

The detection of EVs is based on frequency communication with RFSUs. Then a priority coefficient PC is allocated for each $\mathrm{EV}$ based on the frequency value, where the ambulance and firefighters have the same priority coefficient. Finally, they introduced the priority factor PF, Eq. (2), function of distance $d$, priority coefficient PC and some empirical factors:

$$
\mathrm{PF}=10 \mathrm{PC} \times e^{-0.004 d} \text {. }
$$

Then, the values of the priority factor are classified into three ranges called access categories which determine 
whether the type of required access (very fast, fast, slow) and consequently whether the priority is (first, second or third) priority.

\subsection{Lessons learned}

The work presented by Chowdhury (2016) is one of the most relevant works, in fact, the function in Eq. (2) gives the required aspect of the variation of the priority factor based on the distance using the exponential function; when the distance to the intersection is small, it becomes more urgent to act so the PF is really high. However, some limitations are accorded to Eq. (2), for example it is only based on distance, while in real life the problem is more complex and it should take into consideration the condition of the intersection; how many cars are present, the presence of a traffic jam.

Many inconveniences are present within the presented work of Kapileswar et al. (2017). On one hand, turning lights to green the moment the EV is leaving its location is not advisable as the vehicle may take long time to arrive while other vehicles could have been served during that time and the maximum red time can be violated in this case. On the other hand, the OD of the EV is not always known as the driver starts on a rush and can forget to send its destination, besides, for reasons of security and confidentiality, the police do not mention its OD. Moreover, the types of incidents in real life are more complicated and it is not possible to restrict them in the 3 scenarios as mentioned in the paper

\section{Results and discussions}

\subsection{Priority indicator calculation}

The objective is to create the Priority Indicator (PI) function of not only the Priority class, referred to as Prio $[1,14]$ but also the variation of the (Estimated Time of Arrival) ETA of the EV, the number of cars present in front of the $\mathrm{EV}$ and the time to dissipate this queue $\left(t_{d}\right)$.

- The smaller the ETA the higher the PI becomes because: if the emergency vehicle becomes nearer to the intersection then it is more crucial to act.

- The greater the $t_{d}$ the higher the PI: the more time taken to dissipate the queue, the more likely the emergency vehicle to be delayed

- The higher the prio, the higher the PI: with similar conditions of $t_{d}$ and ETA, vehicles with prio equal to 14 have higher priority than vehicles with prio 13 .

- The exponential function is the best function to introduce the PI, the steepness of the increase and decrease of the priority indicator is important because as it shows the sensitivity towards the variation of the $\mathrm{x}$ factor.

Based on the related works studied previously in part 4, the Eq. (2) will be taken into consideration, thus the priority indicator (PI) is going to be under form in Eq. (3) as follows:

$\mathrm{PI}=c e^{-b x}$.

On one hand, if we take into consideration the variability in time of the priority indicator, we will remark that both the ETA and the $t_{d}$ are constantly variable so, using them in a separated way is not feasible. On the other hand, looking at the priority indicator for vehicles having the same priority class (prio is the same) then the priority indicator is function of only ETA and $t_{d}$. As a result, a link between these two factors is required, if we look at the previously mentioned constraints of the ETA, the $t_{d}$ and the characteristics of the variability of the exponential function we can deduce that:

$x=f\left(\mathrm{ETA}, t_{d}\right)$

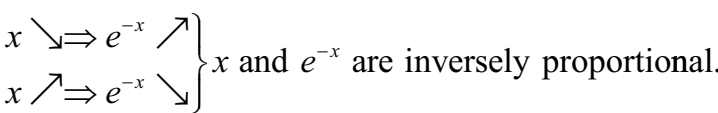

If we study the logical influence of the variation of ETA $-t_{d}$ on PI if prio = constant:

- $\mathrm{ETA}-t_{d} \searrow \Rightarrow \mathrm{PI} \nearrow$ : if it takes approximately the same time to clear the queue of cars in front of the EV and the arrival then the PI will increase, meaning that it's a crucial state and need to be taken into consideration to be served first.

- $\mathrm{ETA}-t_{d} \nearrow \Rightarrow \mathrm{PI} \searrow$ : if the EV is far away in time and only few cars are present in front of it, then the priority of that EV is important but not that crucial as it is easy to deal with.

Consequently:

$x=\mathrm{ETA}-t_{d}$.

Now for the same ETA $-t_{d}$, the priority class with prio 14 need to have higher PI:

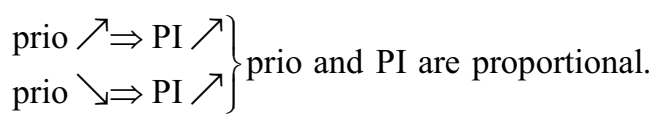

Taking into consideration the previous studies of the variability of the factors and their influences on the priority indicator results in concluding the variable $x$ and the constants and by replacing them in the Eq. (3) we have the form of the priority indicator as mentioned in Eq. (4): 


$$
\mathrm{PI}=a \cdot \operatorname{prio} \cdot e^{-b\left(\mathrm{ETA}-t_{d}\right)} .
$$

Where $a$ and $b$ are constant factors that are determined after.

\subsection{Time to dissipate the queue $\left(t_{d}\right)$}

The time to dissipate the queue is different from one OD to another and is function of several circumstances. It is mainly based on the number of vehicles present in a specific OD or in an arm of the intersection. Thus, having the information of the situation of the previous intersections feeding the studied one, is of a big help in order to have a better estimation of the number of coming vehicles, which can lead to a more accurate calculation of the time needed to clear the way in front of the EV.

Based on the study of historic data of the SPaT 2 of the intersection 201234 in north-Holland equipped with the iVRI, a variation of the number of vehicles departed from the stop line detectors in function of the time it has been green is provided in Fig. 2.

A regression analysis using a second-degree polynomial regression is implemented in order to determine the relationship of the number of departed vehicles $(n d v)$ and the time it has been green $\left(t_{g}\right)$. As a result, the regression's equation helps calculate the time needed to dissipate a given number of cars.

The calculated regression with Octave gave the Eq. (5):

$n d v=-0.0013326 t_{g}^{2}+0.3268624 t_{g}+1.4217784$.

Since the green time $\left(t_{g}\right)$ is the time needed to dissipate a given number of cars $(N)$, then the green time can be replaced by the time to dissipate the queue $\left(t_{d}\right)$ and the number of departed vehicles $(n d v)$ can be replaced by $(N)$, a

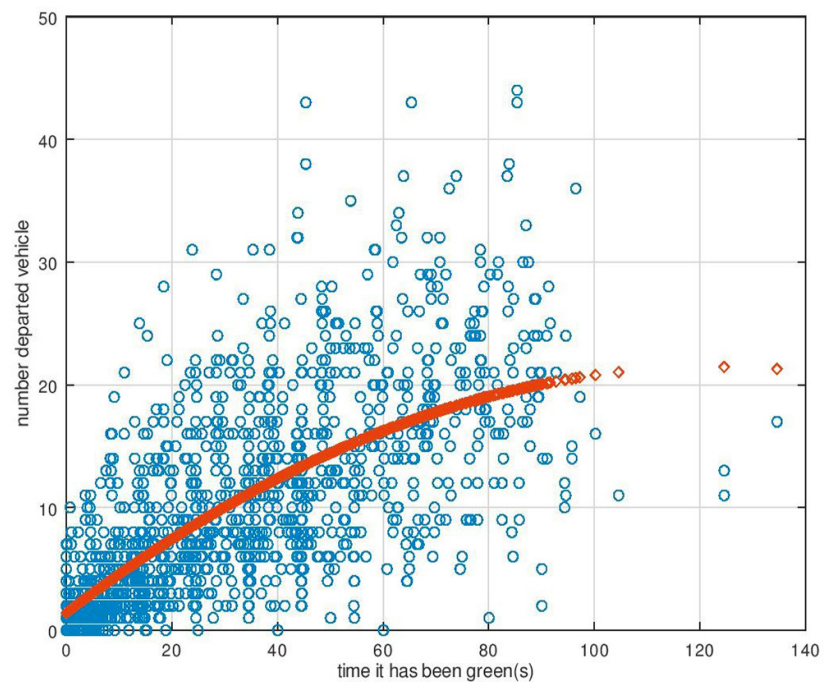

Fig. 2 Departed vehicles function of green time given number of vehicles. Thus, the replacements in Eq. (5) provide us Eq. (6):

$$
-0.0013326 t_{d}^{2}+0.3268624 t_{d}+1.4217784-N=0 .
$$

The Eq. (6) will be used in order to calculate the time to dissipate the queue in this paper, yet the calculation method might be improved by using a machine learning aiming to estimate a more accurate and real-time $t_{d}$.

\subsection{Test of the PI}

This part is designated to test the performance of Eq. (7) of the Priority Indicator (PI) through three crucial examples, thus the Eq. (4) shall be used where $b=0.4, a=10$ :

$$
\mathrm{PI}=10 \cdot \operatorname{prio} \cdot e^{-0.4\left(\mathrm{ETA}^{-t_{d}}\right)} .
$$

\subsubsection{Scenario1: same ETA, same $\boldsymbol{t}_{d}$, different prio}

In this scenario we will examine how the PI will help give priority to two different vehicles where their ETAs are the same and their $t_{d} \mathrm{~s}$ are the same while one has higher prio than the other as summarized in Table 1.

Thus, the calculation of the PI1 and PI2 respectively for EV1 and EV2 based on the information in Table 1 are shown in Table 2.

In this scenario, we assumed there are same number of cars in both section of roads from which the EVs are coming, moreover the two EVs are estimated to arrive in the same time to the intersection, which gave the variation of $t_{d}$ is constant and ETA- $t_{d}$ is constant.

From Table 2, we conclude that the priority indicator for the EV with higher prio factor is constantly higher than EV with lower prio factor if the ETA- $t_{d}$ is constant. Consequently, given same ETA and same $t_{d}$ with same evolution, gives that the EV with higher prio gets priority.

Table 1 Details of the studied EVs

\begin{tabular}{lccc}
\hline & ETA & $t_{d}$ & prio \\
\hline EV1 & 30 & 20 & 13 \\
EV2 & 30 & 20 & 14 \\
\hline
\end{tabular}

Table 2 Calculation of PIs of scenario 1

\begin{tabular}{lcccc}
\hline ETA & $t_{d}$ & ETA- $t_{d}$ & PI 1 & PI 2 \\
\hline 30 & 20 & 10 & 2.38 & 2.56 \\
29 & 19 & 10 & 2.38 & 2.56 \\
28 & 18 & 10 & 2.38 & 2.56 \\
$:$ & $:$ & $:$ & $:$ & $:$ \\
$:$ & $:$ & $:$ & $:$ & $:$ \\
11 & 1 & 10 & 2.38 & 2.56 \\
10 & 0 & 10 & 2.38 & 2.56 \\
\hline
\end{tabular}




\subsubsection{Scenario 2: same ETA, different variation of $\boldsymbol{t}_{d}$, different prio}

In this second scenario, we examine how the PI will help give priority to two different vehicles where their ETAs are the same. However, the traffic conditions in the road's sections are different; one is facing congestion, which means $t_{d}$ is increasing while the other is facing an easygoing flow, no congestion and no delays, which means $t_{d}$ is decreasing. The starting conditions are summarized in Table 3.

Thus, the calculation of the different PIs based on the information in Table 3 are shown in Table 4, where PI1 and PI 2 represent the priority indicator for EV1 and EV2 respectively.

This example aims to see how the PI would react if we have an EV with lower prio factor but with issues of delays because of congestion that increases the time to dissipate the queue (EV2) and another EV with higher prio factor but no problems of congestion (EV1). As mentioned in Table 4, EV1, still 20s to arrive to the intersection and the way is already free. Assuming that the EVs and the flow of the platoons in front of the EVs are maintaining constant speeds, as the congestion is increasing in the case of EV2, then a green extension is required in order to dissipate the queue and let the EV2 leave the intersection.

Thus, the PI2 steadily increases as the $t_{d}$ increases, making it crucial to serve EV2 first.

In order to understand how the priority factor responds to small changes that may cause delay, a zoomed picture is taken for the variation of the PIs of the previous EVs based on the previously mentioned results in Table 4 and detailed conditions of Table 3 . Hence, this results in Fig. 3, it shows how the different variations of the time to dissipate the queue of two different EVs can influence the priority allocation.

As derived from the plot, the PI is function of ETA- $t_{d}$, the smaller this difference is, the more crucial the priority allocation is, thus, the higher the PI.

$$
\begin{array}{cc}
t_{d 1} \searrow & t_{d 2} \nearrow \\
\mathrm{ETA}-t_{d 1} \nearrow & \mathrm{ETA}-t_{d 2} \searrow \\
\mathrm{PI} 1 \searrow & \mathrm{PI} 2 \nearrow
\end{array}
$$

When the ETA- $t_{d}$ is rather big, the traffic lights are

\begin{tabular}{|c|c|c|c|c|c|c|}
\hline & \multicolumn{2}{|r|}{ ETA } & \multicolumn{3}{|c|}{$t_{d}$} & Prio \\
\hline \multicolumn{2}{|l|}{ EV1 } & 30 & \multicolumn{2}{|l|}{20} & $t_{d 1} \downarrow$ & 14 \\
\hline \multicolumn{2}{|l|}{ EV2 } & 30 & \multicolumn{2}{|l|}{20} & $t_{d 2} \uparrow$ & 13 \\
\hline \multicolumn{7}{|c|}{ Table 4 PIs calculations for scenario 2} \\
\hline \multirow[b]{2}{*}{ ETA } & \multicolumn{3}{|c|}{ EV1 (prio14) } & \multicolumn{3}{|c|}{ EV2 (prio13) } \\
\hline & $t_{d 1} \downarrow$ & ETA- $t_{d 1}$ & PI 1 & $t_{d 2} \uparrow$ & ETA- $t_{d 2}$ & PI 2 \\
\hline 30 & 20 & 10 & 2.5641 & 12 & 18 & 0.097056 \\
\hline 29 & 18 & 11 & 1.7188 & 14 & 15 & 0.322238 \\
\hline 28 & 16 & 12 & 1.1521 & 16 & 12 & 1.069867 \\
\hline 27 & 14 & 13 & 0.7723 & 18 & 9 & 3.552084 \\
\hline 26 & 12 & 14 & 0.5177 & 20 & 6 & 11.79333 \\
\hline 25 & 10 & 15 & 0.3470 & 22 & 3 & 39.15525 \\
\hline 24 & 8 & 16 & 0.2326 & 24 & 0 & 130 \\
\hline
\end{tabular}
working normally, and no priority is given. This assures that the circulation is realized in a smooth normal way. The difference is then seen between the two plots when the ETA- $t_{d}$ becomes slight.
Table 3 Detailed information of studied EVs scenario 2

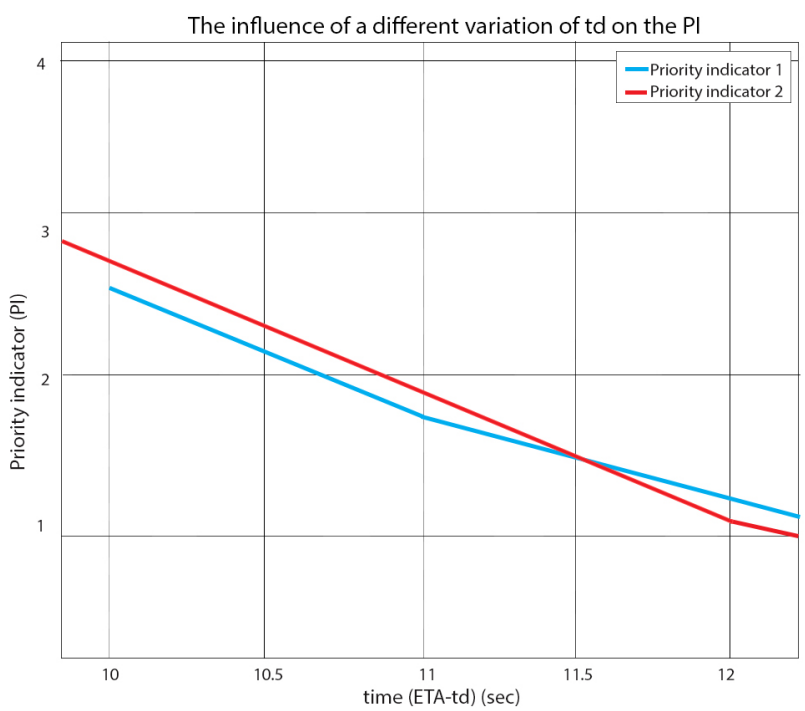

Fig. 3 Variation of PIs based on ETA-td for two different EVs

\subsection{Discussion and implementation scheme}

The study of the PIs allows dealing with several different scenarios of emergency vehicles and aiming to give priority based on the situation. The implementation of the PI in DIRECTOR, which updates every $10 \mathrm{sec}$. In order to achieve optimal traffic light control to provide clearance for emergency vehicles and to shorten their travel time, we propose a time-priority based algorithm with all possible scenarios where not only one EV per direction is involved and the number of EVs can change in time. Each time an $\mathrm{EV}$ is detected driving towards an intersection, it is sorted in the FIFO (First In First Out) list that need to be served in $10 \mathrm{sec}$ then the update allows to rearrange the EVs in the list according to the variation of the calculated PIs. The scheme presented in Fig. 4. 


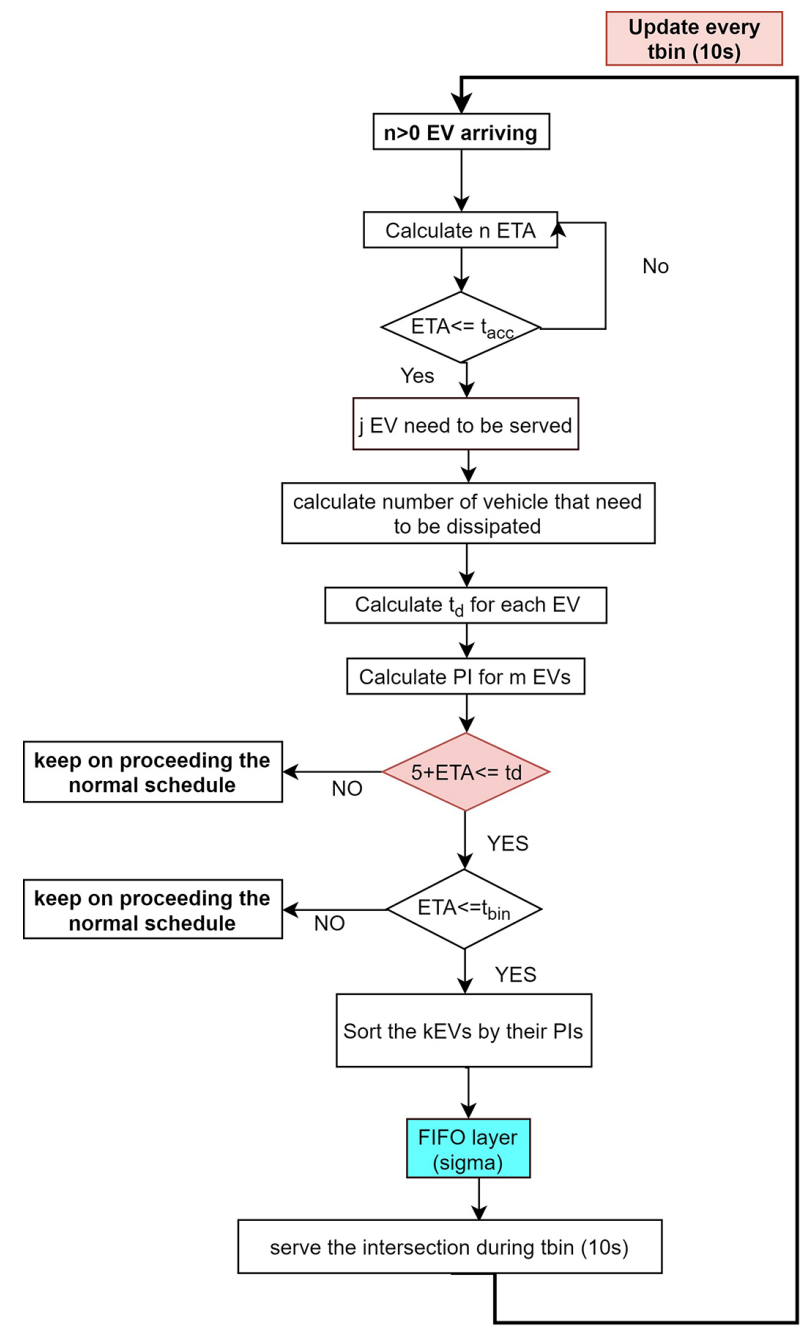

Fig. 4 Scheme of implementation of PI in DIRECTOR

\section{References}

Bigazzi, A. Y., Figliozzi, M. A. (2013) "Marginal costs of freeway traffic congestion with on-road pollution exposure externality", Transportation Research Part A: Policy and Practice, 57, 12-24. https://doi.org/10.1016/j.tra.2013.09.008

Chowdhury, A. (2016) "Priority based and secured traffic management system for emergency vehicle using IoT", In: 2016 International Conference on Engineering \& MIS (ICEMIS), Agadir, Morocco, pp. $1-6$.

https://doi.org/10.1109/ICEMIS.2016.7745309

iVRI viewer "Intelligente Verkeersregelinstallaties in Nederland" (Intelligent Traffic Control Systems in the Netherlands), (in Dutch) [online] Available at: https://www.ivriviewer.nl/ [Accessed: 20 July 2018]

Helmy, N. (2017) "An intelligent traffic flow progression model for predictive control applications", MSc Thesis, Delft University of Technology.

Kapileswar, N., Santhi, P. V., Chenchela, V. K. R., Prasad, C. H. V. S. (2017) "A fast information dissemination system for emergency services over vehicular ad hoc networks", In: 2017 International Conference on Energy, Communication, Data Analytics and Soft Computing (ICECDS), Chennai, India, pp. 236-241. https://doi.org/10.1109/ICECDS.2017.8389862

\section{Conclusion}

Dealing with the traffic mix in an efficient way in signalized intersections requires the implementation of the priority in the traffic controllers taking into consideration all the possible scenarios and assuring that the traffic is as smooth and safe as possible.

This research presents the scheme of the integration of granting absolute priority, priority for emergency vehicles, through the priority indicator and using mathematical approaches in the predictive traffic actuation program DIRECTOR. The realized work deals with the different complex scenarios and assures that the priority is given in the safest way possible.

The priority indicator presented in this work shall be used with other criteria for the implementation of the conditional priority and the scheme can also be adjusted for the general case of the traffic mix.

\section{Acknowledgement}

The project presented in this article was realized within Siemens Mobility Zoetermeer (SNLM), in the aim of improving the program DIRECTOR.

Logghe, S., de Meulenaere, I., Verhoeven, E., Hamelynck, T. (2017) "Denk mee over eenduidig configureren van prioriteit iVRI's" (Think along configuring priorities for iVRI's). (in Dutch)

Nellore, K., Hancke, G. P. (2016) "Traffic Management for Emergency Vehicle Priority Based on Visual Sensing", Sensors, 16(11), Article ID: 1892 .

https://doi.org/10.3390/s16111892

van Senden, J. C. (2018) "DIRECTOR: Enabling advanced driver assistance systems with predictive signalized intersection control using LSTM networks", MSc Thesis, Delft University of Technology.

Viriyasitavat, W., Tonguz, O. K. (2012) "Priority Management of Emergency Vehicles at Intersections Using Self-Organized Traffic Control", In: 2012 IEEE Vehicular Technology Conference (VTC Fall), Quebec City, QC, Canada, pp. 1-4. https://doi.org/10.1109/VTCFall.2012.6399201

Vlahogianni, E. I., Karlaftis, M. G., Golias, J. C. (2014) "Short-term traffic forecasting: Where we are and where we're going", Transportation Research Part C: Emerging Technologies, 43(1), 3-19. https://doi.org/10.1016/j.trc.2014.01.005 